\title{
C- and L-band erbium-doped waveguide lasers with wafer-scale silicon nitride cavities
}

\author{
Purnawirman, ${ }^{1, *}$ J. Sun, ${ }^{1}$ T. N. Adam, ${ }^{2}$ G. Leake, ${ }^{2}$ D. Coolbaugh, ${ }^{2}$ J. D. B. Bradley, ${ }^{1}$ \\ E. Shah Hosseini, ${ }^{1}$ and M. R. Watts ${ }^{1}$ \\ ${ }^{1}$ Photonic Microsystems Group, Research Laboratory of Electronics, Massachusetts Institute of Technology, \\ 77 Massachusetts Avenue, Cambridge, Massachusetts 02139, USA \\ ${ }^{2}$ College of Nanoscale Science and Engineering, University at Albany, State University of New York, \\ 257 Fuller Road, Albany, New York 12203, USA \\ ${ }^{*}$ Corresponding author: purna@mit.edu
}

Received March 18, 2013; revised April 16, 2013; accepted April 21, 2013;

posted April 22, 2013 (Doc. ID 187023); published May 20, 2013

\begin{abstract}
We report on integrated erbium-doped waveguide lasers designed for silicon photonic systems. The distributed Bragg reflector laser cavities consist of silicon nitride waveguide and grating features defined by wafer-scale immersion lithography and a top erbium-doped aluminum oxide layer deposited as the final step in the fabrication process. The resulting inverted ridge waveguide yields high optical intensity overlap with the active medium for both the $0.98 \mu \mathrm{m}$ pump $(89 \%)$ and $1.5 \mu \mathrm{m}$ laser $(87 \%)$ wavelengths with a pump-laser intensity overlap of $>93 \%$. We obtain output powers of up to $5 \mathrm{~mW}$ and show lasing at widely spaced wavelengths within both the $\mathrm{C}$ and $\mathrm{L}$ bands of the erbium gain spectrum $(1536,1561$, and $1596 \mathrm{~nm})$. @ $(2013$ Optical Society of America

OCIS codes: (130.0130) Integrated optics; (130.2790) Guided waves; (130.3120) Integrated optics devices; (140.3460) Lasers.

http://dx.doi.org/10.1364/OL.38.001760
\end{abstract}

Integrated lasers in a silicon photonics platform have been intensively researched because silicon itself is a poor light emitter [1]. Several methods have been applied to realize integrated lasers, including germanium-onsilicon heterojunctions [2], hybrid integration with III-V semiconductor materials [3] , stimulated Raman scattering [4] ], and erbium-doped glass on silicon [5]. Of these approaches, only erbium-doped glass lasers have the advantage of a straightforward, monolithic fabrication process while yielding high-performance narrow-linewidth lasers. In particular, erbium-doped aluminum oxide $\left(\mathrm{Al}_{2} \mathrm{O}_{3}: \mathrm{Er}^{3+}\right)$ has been cosputtered onto oxidized silicon wafers with relatively low loss and a broad gain spectrum [6], resulting in racetrack [7] and ultranarrow-linewidth distributed feedback lasers []ㅡ. However, to define the laser waveguide and cavity, previous works used interference lithography [] $]$ and etching of the gain material $[\underline{7}, 8]$, both of which are difficult to incorporate within standard waferscale silicon photonics process flows. A preferable approach would be to define laser cavities using standard complementary metal-oxide-semiconductor (CMOS) processing techniques while reducing unnecessary fabrication steps on the erbium-doped layer.

Alternatively, silicon nitride $\left(\mathrm{SiN}_{x}\right)$ is a low-loss, frontend of line, CMOS compatible material [9] that can be used in the formation of high-resolution photonic features, including waveguides, gratings, and even laser cavities. An erbium-doped glass layer can then be deposited as a backend process step without further etching or processing required. This approach enables large-scale production of erbium-doped waveguide lasers and integration with silicon nitride passive components on silicon photonic chips.

In this Letter, we demonstrate integrated $\mathrm{Al}_{2} \mathrm{O}_{3}: \mathrm{Er}^{3+}$ lasers with high-resolution $\mathrm{SiN}_{x}$ cavities patterned in a state-of-the-art $300 \mathrm{~mm}$ silicon wafer line. We obtain more than $5 \mathrm{~mW}$ of optical power from a distributed Bragg reflector (DBR) cavity at $1561 \mathrm{~nm}$. In addition, we observe laser output over a wide wavelength range $(1536,1561$, and $1596 \mathrm{~nm}$ ), demonstrating the possibility to integrate laser sources across both the C (1530-1570 nm) and L (1570-1625 nm) bands with silicon photonics technology.

One of the main challenges in the waveguide design is the relatively high refractive index contrast of $\mathrm{SiN}_{x}$ $(n=1.99)$ and $\mathrm{Al}_{2} \mathrm{O}_{3}: \mathrm{Er}^{3+}(n=1.65)$. The intensity distribution of the fundamental transverse-electrical (TE) mode tends to be more confined in the high-index material, especially for shorter wavelengths. This trend is contrary to the design approach for an efficient erbium-doped laser waveguide, where high confinement in the active material and high intensity overlap between the pump ( 980 nm) and signal $(\sim 1550 \mathrm{~nm})$ modes are preferred. Furthermore, any additional optical intensity localized in the $\mathrm{SiN}_{x}$ waveguide may introduce detrimental intracavity losses from scattering and the intrinsic absorption of the material. To overcome this, our waveguide design includes a layer of $\mathrm{SiO}_{2}(t=0.1 \mu \mathrm{m}$, $n=1.44)$ between the $\operatorname{SiN}_{x}$ waveguide $(t=0.1 \mu \mathrm{m}$, $w=4.0 \mu \mathrm{m})$ and the $\mathrm{Al}_{2} \mathrm{O}_{3}: \mathrm{Er}^{3+}$ film $(t=1.4 \mu \mathrm{m})$, as shown in Fig. 1(a). The oxide gap reduces the guiding effect of the high refractive index $\operatorname{SiN}_{x}$ ridge and increases the confinement within the $\mathrm{Al}_{2} \mathrm{O}_{3}: \mathrm{Er}^{3+}$ layer. Figures 1(b) and 1(c) show the intensity distributions of the fundamental TE mode at $980 \mathrm{~nm}$ (pump $p$ ) and
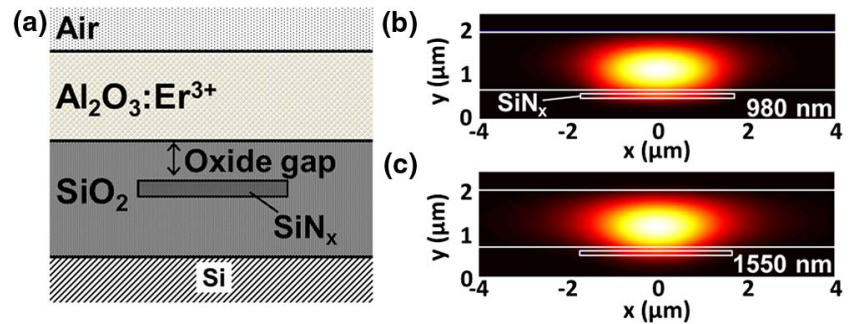

Fig. 1. (a) Cross-sectional view of the laser waveguide structure. The calculated intensity distribution of the fundamental TE mode at (b) 980 and (c) $1550 \mathrm{~nm}$. 
$1550 \mathrm{~nm}$ (signal $s$ ), respectively. If we define the intensity confinement factor $\left(\gamma_{s p p}\right)$ and intensity overlap $\left(\Gamma_{s p}\right)$ by the following,

$$
\begin{gathered}
\gamma_{s / p}=\frac{\int_{A} I_{s / p} \mathrm{~d} A}{\int_{\infty} I_{s / p} \mathrm{~d} A} \approx \frac{\sum_{\text {active }(i j)} I_{i j}^{(s / p)}}{\sum_{i j} I_{i j}^{(s / p)}}, \\
\Gamma_{s p}=\frac{\int_{A} I_{p} I_{s} \mathrm{~d} A}{\sqrt{\int_{\infty} I_{p}^{2} \mathrm{~d} A \int_{\infty} I_{s}^{2} \mathrm{~d} A}} \approx \frac{\sum_{\text {active }(i j)} I_{i j}^{(p)} I_{i j}^{(s)}}{\sqrt{\sum_{i j} I_{i j}^{2(p)}} \sqrt{\sum_{i j} I_{i j}^{2(s)}}},
\end{gathered}
$$

then we obtain $\gamma_{s}=87 \%, \gamma_{p}=89 \%$, and $\Gamma_{s p}=93 \%$. Note that the integration of the mode intensity is approximated by the summation over discrete space coordinates $i$ and $j$. Thus our waveguide design yields high-intensity confinement factors in the active medium and is highly wavelength insensitive, permitting either resonant pumping around $1480 \mathrm{~nm}$ or pumping near $980 \mathrm{~nm}$, where $\mathrm{Yb}$ codoping can be advantageous [10].

The silicon-based integrated laser cavities were fabricated within a $300 \mathrm{~mm}$ line in a standard CMOS foundry. A $6 \mu \mathrm{m}$-thick plasma-enhanced chemical vapor deposition (PECVD) $\mathrm{SiO}_{2}$ layer was grown, followed by deposition of a $0.1 \mu \mathrm{m}$-thick PECVD $\mathrm{SiN}_{x}$ layer. Both PECVD layers were chemically mechanically polished (CMPed) to reduce losses due to surface roughness. The silicon nitride layer was subsequently annealed at $1050^{\circ} \mathrm{C}$ for 72 min to reduce absorption due to $\mathrm{Si}-\mathrm{H}$ and $\mathrm{N}-\mathrm{H}$ bonds around $1.52 \mu \mathrm{m}$ [9]. The nitride layer was then patterned using $193 \mathrm{~nm}$ immersion lithography and reactive ion etching. After patterning, a PECVD $\mathrm{SiO}_{2}$ layer was deposited and CMPed to a final height of $0.1 \mu \mathrm{m}$ above the silicon nitride layer. Trenches for dicing and fiber end coupling were then etched into the edges of the dies by deep oxide and silicon etching. Finally, the wafers were transferred from the CMOS foundry, diced into individual dies, and an $\mathrm{Al}_{2} \mathrm{O}_{3}: \mathrm{Er}^{3+}$ layer was deposited by reactive cosputtering using a process similar to that reported in [11]. Using the prism coupling method to measure the planar losses around $1550 \mathrm{~nm}[6,12]$, we determine the background loss and dopant concentration in the $\mathrm{Al}_{2} \mathrm{O}_{3}: \mathrm{Er}^{3+}$ film to be $<0.1 \mathrm{~dB} / \mathrm{cm}$ and $1.4 \times$ $10^{20} \mathrm{~cm}^{-3}$, respectively.

The DBR resonator consists of a straight waveguide of length $L_{\text {gain }}=20 \mathrm{~mm}$ and width $w=4.0 \mu \mathrm{m}$ confined by two reflection gratings, as shown in Fig. 2(a). The gratings are formed by waveguides of length $d=1.5 \mathrm{~mm}$ with periodically notched sidewalls having notch widths of $s_{1}$ at one end and $s_{2}$ at the other end. $s_{1}$ is fixed at $1.29 \mu \mathrm{m}$ for a reflectivity of $\sim 100 \%$ and $s_{2}$ is varied from 418 to $796 \mathrm{~nm}$ to achieve a reflectivity of $85 \%$ to $99.6 \%$. Figure 2(b) shows the calculated reflectivity of the grating with respect to variation of the sidewall etch by coupled mode theory [13] and an SEM image of one of the right-side gratings. Furthermore, the grating period, $\Lambda$, is varied among lengths of 478,487 , and $498 \mathrm{~nm}$, which correspond to Bragg wavelengths of 1536, 1561, and $1596 \mathrm{~nm}$, respectively.

Figure 3 shows the experimental setup used to measure the lasers. Pump light from 978 and $975 \mathrm{~nm}$ laser diodes is coupled into the chip on each side using fiber

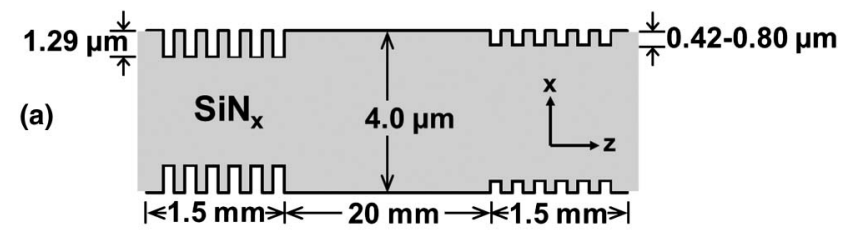

(b)

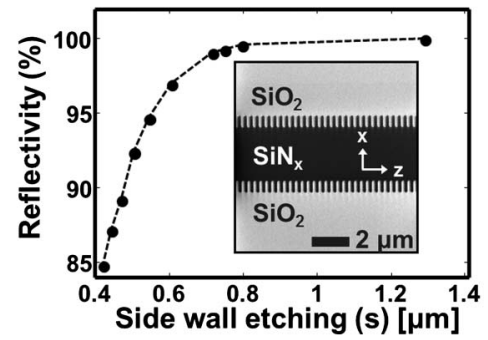

Fig. 2. (a) Top view diagram of the DBR laser cavity. (b) Plot of reflectivity at variation of sidewall etch widths on the chip by coupled mode theory. The small inset shows the SEM image of one of the gratings of the DBR laser.

980/1550 nm wavelength division multiplexers (WDMs), respectively. The laser output is collected from the $1550 \mathrm{~nm}$ port of the WDM and the power is measured using a powermeter while the spectrum is recorded by an optical spectrum analyzer (OSA).

By insertion loss measurements, we estimated coupling losses at each facet of the chip of -5.6 and $-7.3 \mathrm{~dB}$ for 980 and $1550 \mathrm{~nm}$ TE-polarized light, respectively. Using the measured facet loss, we determine launched pump powers of 147 and $98 \mathrm{~mW}$ from 978 and $975 \mathrm{~nm}$ diodes, respectively. The maximum output power is obtained from the DBR laser with center wavelength at $1561 \mathrm{~nm}$ and reflectivities of $R_{1}=100 \%$ and $R_{2}=95 \%$. To characterize the laser performance, we plot the output power at different pump powers, as shown in Fig. 4 . We start by pumping from the $978 \mathrm{~nm}$ diode (left) only. When the diode reaches the maximum pump power, we turn on the pump from the $975 \mathrm{~nm}$ diode (right). We obtain maximum on-chip output power of $P_{\max }=5.1 \mathrm{~mW}$ with a lasing threshold of $P_{\text {th }}=44 \mathrm{~mW}$. This corresponds to a slope efficiency of $\eta=2.6 \%$.

In addition, we obtain maximum output powers for DBRs with different grating periods of $P_{\max }=2.5 \mathrm{~mW}$ $\left(\lambda_{c}=1536 \mathrm{~nm}\right)$ and $P_{\max }=0.5 \mathrm{~mW} \quad\left(\lambda_{c}=1596 \mathrm{~nm}\right)$. These outputs are obtained from cavities with reflectivity of $R_{2}=86 \% \quad\left(\lambda_{c}=1536 \mathrm{~nm}\right) \quad$ and $\quad R_{2}=95 \% \quad\left(\lambda_{c}=\right.$ $1596 \mathrm{~nm}$ ), respectively. The difference in the optimum reflectivity for the DBR cavities can be attributed to the difference in the gain threshold and maximum small

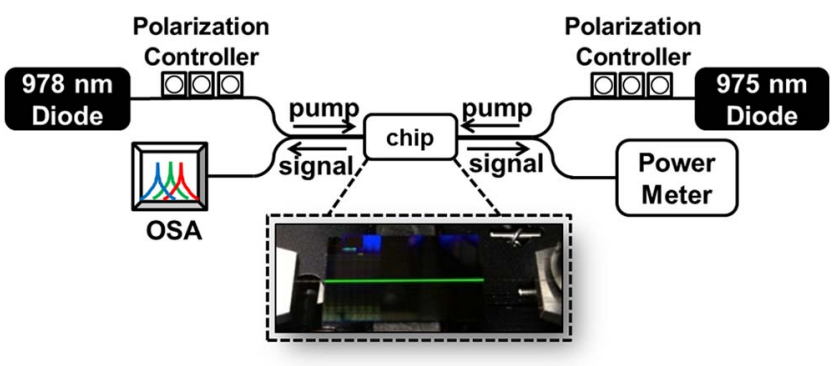

Fig. 3. Experimental setup of the measurement. 


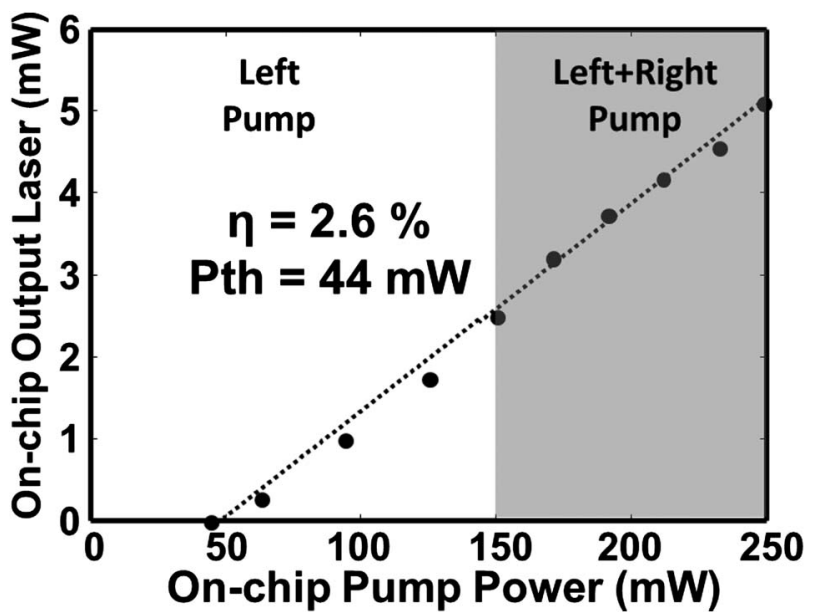

Fig. 4. Output power versus on-chip pump power for the DBR laser at a $1561 \mathrm{~nm}$ laser wavelength.

signal gain at different wavelengths. Figure 5 shows the spectra of three different lasers recorded by the OSA. The laser emission at $1596 \mathrm{~nm}$ is a consequence of low grating scattering loss, low background losses in the $\mathrm{Al}_{2} \mathrm{O}_{3}: \mathrm{Er}^{3+}$ film, and the wide gain spectrum of $\mathrm{Al}_{2} \mathrm{O}_{3}: \mathrm{Er}^{3+}[\underline{6}$. We note that the DBR laser spectra lie within two important communication bands, the $\mathrm{C}$ band and the L band. By simply modifying the period of the gratings in the silicon nitride layer, it is feasible to fabricate arrays of lasers spanning both communication bands.

In summary, we have demonstrated integrated erbiumdoped aluminum oxide lasers with DBR cavities defined in $\mathrm{SiN}_{x}$ layers. The laser cavity design was defined using $193 \mathrm{~nm}$ immersion lithography within a standard CMOS foundry and the novel inverted ridge waveguide design enabled the erbium-doped aluminum oxide layer to be deposited as a final backend-processing step. The waveguide structure is designed to have high confinement factor in the active medium for both the $980 \mathrm{~nm}$ pump wavelength (89\%) and the $1550 \mathrm{~nm}$ laser emission wavelength (87\%), with intensity overlap of $>93 \%$. Maximum output power of $2.5,5.1$, and $0.5 \mathrm{~mW}$ are obtained from DBR cavities at center wavelength at 1536, 1561, and $1596 \mathrm{~nm}$, respectively. These laser wavelengths lie within both the $\mathrm{C}$ and $\mathrm{L}$ bands of the erbium gain spectrum. The main advantage of this approach lies in the robust, highresolution, and wafer-scale silicon nitride structuring and backend deposition of the erbium-doped aluminum oxide layer. More complex structures, such as DFB Bragg cavities can be directly patterned in the $\mathrm{SiN}_{x}$ layer. Furthermore, these structures can be mass produced and integrated with other silicon photonic devices in a standard CMOS foundry.

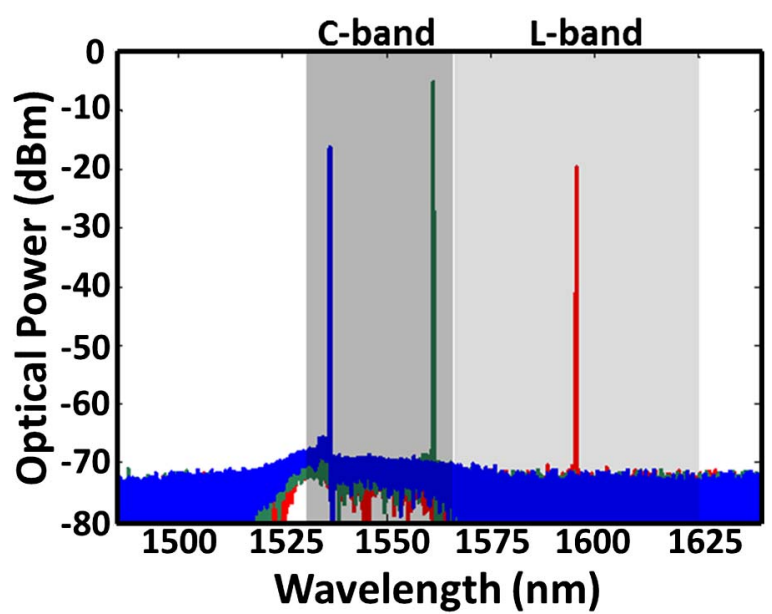

Fig. 5. Spectrum of the output laser within the C- and L- band.

This work is funded by Defense Advanced Research Projects Agency Electronic Photonics Integration (DARPA-EPHI) and program, grant no. HR0011-12-2-0007 and the Samsung Global Research Outreach (GRO) Program.

\section{References}

1. G. T. Reed, Silicon Photonics: The State of the Art (Wiley, 2008), pp. 147-153.

2. R. E. Camacho-Aguilera, Y. Cai, N. Patel, J. T. Bessette, M. Romagnoli, L. C. Kimerling, and J. Michel, Opt. Express 20, 11316 (2012).

3. H. Park, A. W. Fang, S. Kodama, and J. E. Bowers, Opt. Express 13, 9460 (2005).

4. O. Boyraz and B. Jalali, Opt. Express 12, 5269 (2004).

5. T. Kitagawa, K. Hattori, M. Shimizu, Y. Ohmori, and M. Kobayashi, Electron. Lett. 27, 334 (1991).

6. J. D. B. Bradley, L. Agazzi, D. Geskus, F. Ay, K. Wörhoff, and M. Pollnau, J. Opt. Soc. Am. B 27, 187 (2010).

7. J. D. B. Bradley, R. Stoffer, L. Agazzi, F. Ay, K. Wörhoff, and M. Pollnau, Opt. Lett. 35, 73 (2010).

8. E. H. Bernhardi, H. A. G. M. van Wolferen, L. Agazzi, M. R. H. Khan, C. G. H. Roeloffzen, K. Wörhoff, M. Pollnau, and R. M. de Ridder, Opt. Lett. 35, 2394 (2010).

9. N. Sherwood-Droz and M. Lipson, Opt. Express 19, 17758 (2011).

10. P. Laporta, S. Taccheo, S. Longhi, O. Svelto, and C. Svelto, Opt. Mater. (Amsterdam) 11, 269 (1999).

11. K. Wörhoff, J. D. B. Bradley, F. Ay, D. Geskus, T. P. Blauwendraat, and M. Pollnau, IEEE J. Quantum Electron. 45, 454 (2009).

12. P. K. Tien, Appl. Opt. 10, 2395 (1971).

13. T. Murphy, "Design, fabrication, and measurement of integrated Bragg grating optical filters," Ph.D. dissertation (Department of Electrical Engineering and Computer Science, MIT, 2001). 